\title{
Experimental Study on Effect of Laser Hardening Parameters on Carbon Steel, Non-Malleable Cast iron and X20Cr13 Materials
}

\author{
K. Prashanthi ${ }^{1}$, B. Ramakrishna ${ }^{2}$
}

1PG Student, Department of Mechanical Engineering, Chiranjeevi Reddy Institute of Engineering \&

Technology, Anantapur, India

${ }^{2}$ Assistant Professor, Department of Mechanical Engineering, Chiranjeevi Reddy Institute of Engineering \& Technology, Anantapur, Andhra Pradesh, India

\begin{abstract}
Laser hardening is a surface heat treatment process used to enhance tribological and mechanical properties of metals which also leads to increase in service life of the components. Material removal, wear and tear, load concentration occurs mostly at rotating and reciprocating parts. Hence it is sufficient to enhance the hardness of a component at functional areas rather than the entire component. Laser hardening process is designed to change the microstructure of metals through controlled heating and cooling to get a modified surface. The constraints of traditional surface heat treatment process such as inability to treat specific area, distortion, poor degree of controllability, requirement of a quenching medium, long cycle time can be overcome by using Laser surface heat treatment and in addition to that it can be automated. With its benefits Laser surface hardening turns out to be a cost effective and energy saving process. The presented work is an investigation of the laser surface hardening via experimental results making use of a 6 axis robotic arm and a 10KW high power diode laser system as heat source with a wavelength of $980 \mathrm{~nm}$ on leading automotive parts such as retainer, hub, and turbine blade whose materials being non-malleable cast iron, carbon steel, X20Cr13 respectively. Process parameters such as laser power from power source, scan speed were varied to understand the influence on resulting heat treated surface and efforts were made to optimize the process parameters to attain maximum hardness for the component to enhance its working life.
\end{abstract}

Keywords : Laser Hardening, Cast iron, Carbon steel, X20Cr13

\section{INTRODUCTION}

Laser is an acronym for "Light Amplification by Stimulated Emission of Radiation". Exciting atoms to a high energy level and in order to have an inversion in population with respect to an intermediate level is the principle of a laser. When radiation interacts with the excited atom with the frequency that corresponds to the energy difference between the higher energy state of the atom and the intermediate state with inverse population, it produces a radiation with the same phase as the incident radiation. This radiation is amplified with a resonance cavity to get acoherent beam. Another unique property of a laser beam is the high degree of monochromaticity that allows efficient focusing.

Most of the moving parts, that is rotating and reciprocating parts are subjected to wear and tear, which results in power losses in power transmission components such as gears in automobile engines, aircraft engines, steam turbines, gas turbines, power plants, industrial machines, etc. Outer surface of many components are affected by corrosion, which all results in decrease of life of a component. Surface condition is one among the important engineering 
factors of a component. Therefore there originates a need to search for surface treatment in order to save the surface of a component from corrosion, wear and tear. In practice there are a several treatments which deal with a wide range of modified thickness of the components. Choice has to be made carefully while selecting a surface treatment process so that it doesn't affect the bulk properties while we are busy improving the surface properties of the component.

Lasers contribution in the sector of surface engineering applications in the recent few decades is well known. Laser surface modification technique is used in modification of the surface properties and developing wear resistant and corrosion resistant surfaces without affecting the bulk properties of the component. The life of the component is thus seen to be increased by laser surface modification to a considerable extent.

\subsection{REASEARCH OBJECTIVE}

Laser surface hardening which serves as a solution to nonaddition of weight and also rules out the demerits of conventional hardening process.

$>$ The objective of this project was to improve the surface hardness of Carbon Steel, Non Malleable Cast Iron and X20Cr13 materials by laser heat treatment using diode laser.

$>$ To determine optimum laser hardening parameters to maximize hardness above 45 HRC till a case depth of minimum $0.5 \mathrm{~mm}$ with less power consumption.

$>$ Thus increase the life of the components.

\section{LITERATURE REVIEW}

> S. Balasubramanian, V. Muthukumaran and P.Satyabalan," AStudy on theEffect of Process Parameters of Laser Hardening Carbon Steels[2017]".
Babic M, Balic J, Milfelner M, Belic I, Kokol P, Zorman Robot Laser Hardening and the Problem of Overlapping Laser Beam [2013].

MatejBabic, MatjaMilfelner, Igor Beli, PeterKokol Problems Associated with a Robot Laser Cell used for Hardening [2012].

\section{SURFACE TRANSFORMATION USING LASER}

\subsection{HIGH POWER DIODE LASERs [HPDLs]}

The first application of a diode laser in changing the surface properties of a material was carried out in 1991[20]. In recent days diode laser with continuous wave laser output and with laser power output greater than $0.5 \mathrm{~W}$ (class IV) are categorized into High Power Diode Laser sections. Diode lasers overcome the drawbacks of most popular $\mathrm{CO} 2$ lasers and Nd:YAG lasers, in the sectors such as precision of laser process control, power consumption, efficiency and more over high power diode laser application are more economical in comparison to other types of laser.

The $\mathrm{p}-\mathrm{n}$ junction of a diode is used to convert the electrical energy and transform it into laser beam that is light energy which in turn heats up the material converting it into heat energy. Usually InGaAs/GaAs $\mathrm{p}-\mathrm{n}$ junction diodes produce light in the wavelengths of $808 \mathrm{~nm}$ and in case of $\mathrm{In}-\mathrm{GaAlAs} / \mathrm{GaAs}$ p-n junction diodes producing light in the wavelength of $940 \mathrm{~nm}$, two slabs of such semiconductor diodes are constructed to carry out this process. A lot of heat will be generated during the process of energy conversion using $\mathrm{p}-\mathrm{n}$ junction diodes, which can impact the efficiency of the overall process. 


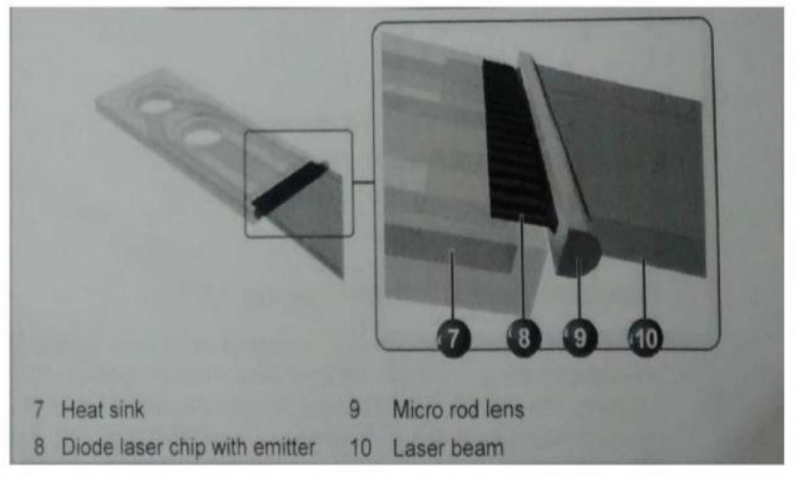

Fig 1. Priciples of Diode Laser Bars

The HPDL bars can further be stacked together in order to generate an increased laser power up to 1 KW [19] and such a process of stacking HPDL bars is called as Diode Stack. To further enhance the laser power this diode stacks can be combined with the help of dielectric mirrors helping to set the same optical pathway for all laser rays generated. Demineralized (DM) water is used to disperse the heat generated during this process. DM water sets an inner cooling circuit that absorbs the heat generated by diode stack through heat sinks and carries the absorbed heat to the external cooling circuits and transfers the heat to it. Thus keeping the, laser power generation section always intact, without overheating and giving rise for a chance of reduction in process efficiency.

\subsection{CHARACTERISTICS OF HPDLS}

\subsubsection{WAVELENGTH}

During the operation of laser the major factor that impacts the process is the reflectivity of the material. The more is the reflection, the poorer, the process results. The better is the absorption the higher the process perfection. Fig 3.6 shows the relationship between reflectivity and the wavelength of incident light. It is seen that the reflection of $\mathrm{CO} 2$ laser and Nd:YAG are much higher in comparison to HPDL's.CO2 laser emits laser beam at $10.6 \mu \mathrm{m}$ and $\mathrm{Nd}$ :YAG laser emits laser beam at $1.06 \mu \mathrm{m}$ whereas HPDL's emits laser beams at $0.82 \mu \mathrm{m}$, which serves higher absorption rate in comparison to $\mathrm{CO} 2$ and Nd:YAG lasers. The wavelengths of HPDL's are in the range of $800-940 \mu \mathrm{m}$ which is minimal than the usual major laser applicants, CO2and Nd:YAG lasers.

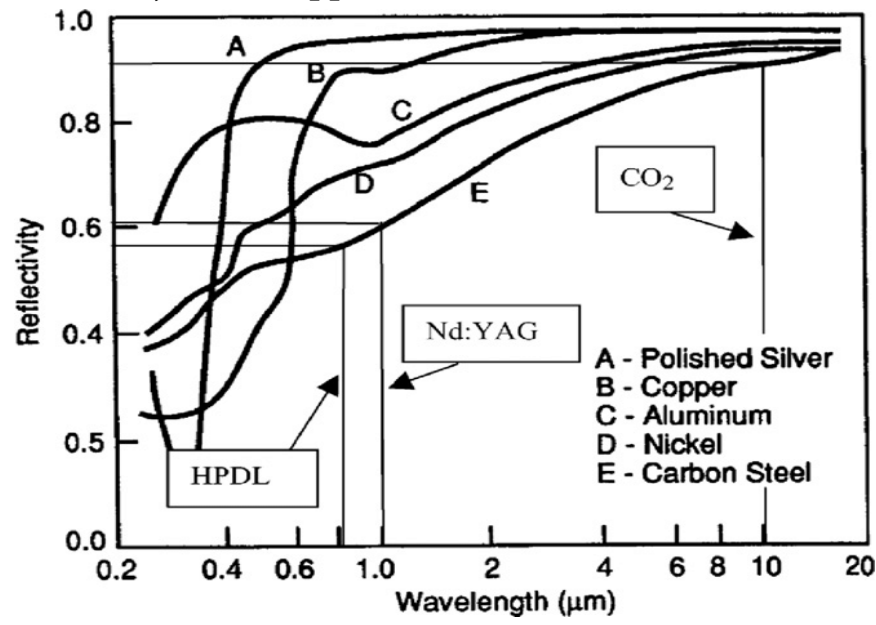

Fig 2. Reflectivity as a function of Wavelength for different materials

\subsubsection{BEAM CHARACTERISTICS}

The CO2 and Nd:YAG lasers are in the capability of generating a single laser source beam, with which a minute change or variation during the excitement of laser in active medium results in intensity fluctuations seen as spike irregularities in the beam profile. On the other hand HPDL's produce a number of laser beams. It's the major difference between other lasers and HPDL's this huge number of laser beams solves the spike irregularities problem by superimposing the huge number of laser beams which results in a modal stabilized beam source.

\subsection{LASER HARDENING OVER CONVENTIONAL HARDENING AND SURFACE IMPROVEMENT TECHNIQUES}

Compared to the conventional hardening and surface improvement techniques, laser hardening overcomes most of the demerits and emerges out with a lot of additional process merits. In conventional process like induction hardening, flame hardening, etc. usually results in high rate of thermal distortion. But laser hardening provides benefits of localized heat input with which thermal distortion can be reduced as the laser hardening process treats the surface with 
minimum energy input and mainly on the surface rather than the entire component. Due to this property laser hardening can be used to treat specific areas and complex shapes and structures. Since laser beams alter only the top surface the internal hardness of the component is not compromised. In addition to that the remaining surface acts as a cooling medium and helps to cool the top surface giving rise to martensite layer at the surface. Laser hardening process can be automated and computerized with which the accuracy, precision and repeatability and quality increases.

The principle of laser hardening is similar to conventional hardening that is to heat the surface above critical temperature, so that the structure is altered and to bring down the temperature rapidly to hold the new structure at room temperature. Laser hardening is a solid state transformation where material is heated below its melting temperature that is till recrystallization temperature and rapidly cooling down before the carbon present starts to precipitate into the cementite phase.

Austenite is a high temperature phase of iron at elevated temperature a gamma phase of iron, a solid solution of iron and alloying elements. When Austenite is rapidly cooled it gives rise to a nonequilibrium martensite phase, formed due to the diffusion less phase transformation. Martensite is brittle and harder with the formation of Martensite structure the wear resistance of the material at surface is thus enhanced. With rapid cooling of carbon steels at austenite form leaves carbon atoms with no time to diffuse out of crystal structure in large enough quantities to form cementite(Fe3C).

In order to get a martensite structure a high intensity laser beam is made to pass on the material as shown in fig3. The high intensity laser beam heats the surface of workpiece and takes it to the austenite phaseat elevated temperatures. But the laser hardening increases only the temperature of the surface whereas the temperature of remaining part of the component is much cooler and is far below the high temperature at the surface. This temperature difference leads to the difference in temperature gradient. This so formed temperature gradient leads to rapid cooling of the surface by heat transferred to the remaining component part through conduction and some amount of heat transferred to the much cooler surrounding through convection. This rapid cooling in turn leads to the formation of martensite region. This way laser hardening has no need of external cooling aid and is a self-quenching process.

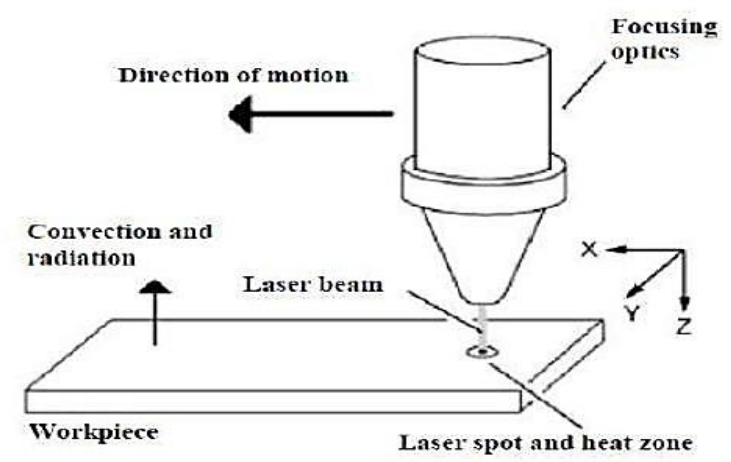

Fig 3. Basic setup of Laser Hardening of a flat plate work piece

\section{EXPERIMENTAL PROCEDURE}

\subsection{SAMPLE MATERIALS}

The three different materials considered here are X20Cr13, Non-malleable Cast Iron and Carbon Steel. The details about chemical composition, size, application, hardness are discussed below.

\subsubsection{STEAM TURBINE BLADE}

Steam Turbine blade is the component of X20Cr13 material considered for the laser surface hardening analysis as shown in fig 4.1. X20Cr13 is a kind of hot forged steel where X20 indicates the parts of carbon present per 10000 parts and Cr13 indicates the percentage of chromium. Due to pressure variation in 
steam turbine region, material removal occurs at the edges of steam turbine blades. The material has a hardness of 28HRC before hardening.So, there arises a need to increase the hardness in order protect the component from pitting action and to increase the life of the component.

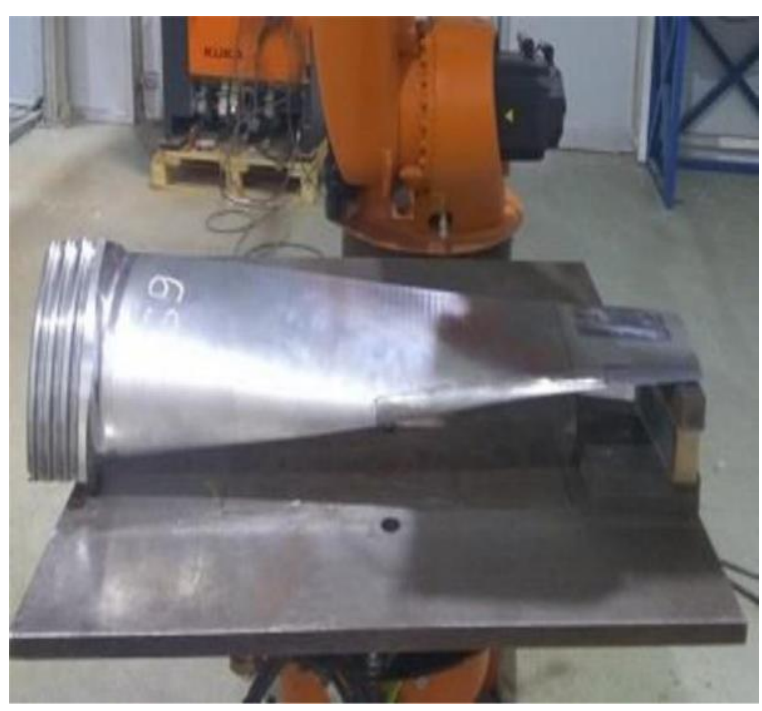

Fig 4. X20Cr13 material

\subsubsection{RETAINER}

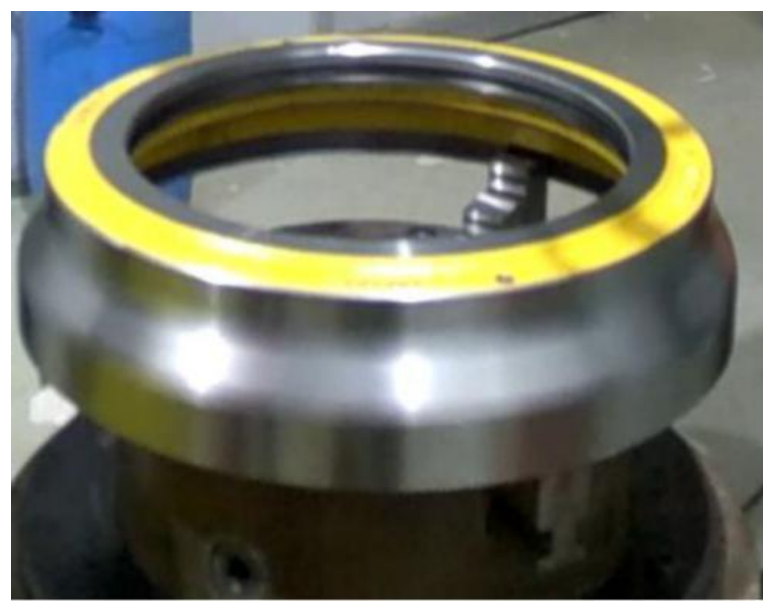

Fig 4.2 : Non-malleable Cast Iron material

Retainer is the component with non-malleable cast iron material. Retainer serves the purpose of shielding the rotating parts in earth mover automotive machines as shown in fig 4.2. Material removal occurs at top region which comes in contact with other rotating parts and causes wear and tear between rotating parts which results in decreasing the life of the component.The material hardness is 26 HRC prior to laser surface hardening. The regions where the surface properties are to be enhanced are top surface with inner diameter of $290 \mathrm{~mm}$ to outer diameter $330 \mathrm{~mm}$ of the component.

\subsubsection{HUB}

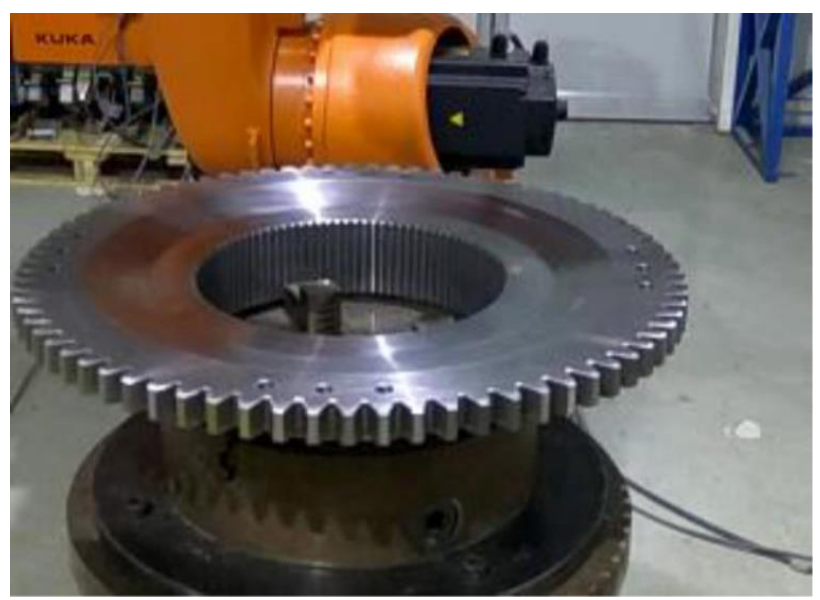

Fig 4.3 : Carbon Steel material

Hub is the component with Carbon Steel EN24 material shown in fig 4.3. Hub serves as a power transmission component in one of the leading earth moving automotive machines. The top and bottom region of hub coming in contact with other parts of shaft results in wear and tear of surface. So, there arises a need to increase the hardness of this region with inner diameter of $250 \mathrm{~mm}$ and outer diameter of $290 \mathrm{~mm}$. The material has a hardness of $24 \mathrm{HRC}$ prior to laser surface hardening.

\subsection{EXPERIMENTAL SETUP}

In this study a $10 \mathrm{KW}$ high power diode laser manufactured from Laserline Company is utilized as shown in fig 4.4. This laser produces a laser beam of with a wavelength ranging from 980-1027 $\mathrm{nm}$ and beam quality of $40 \mathrm{~mm}$.mrad. 


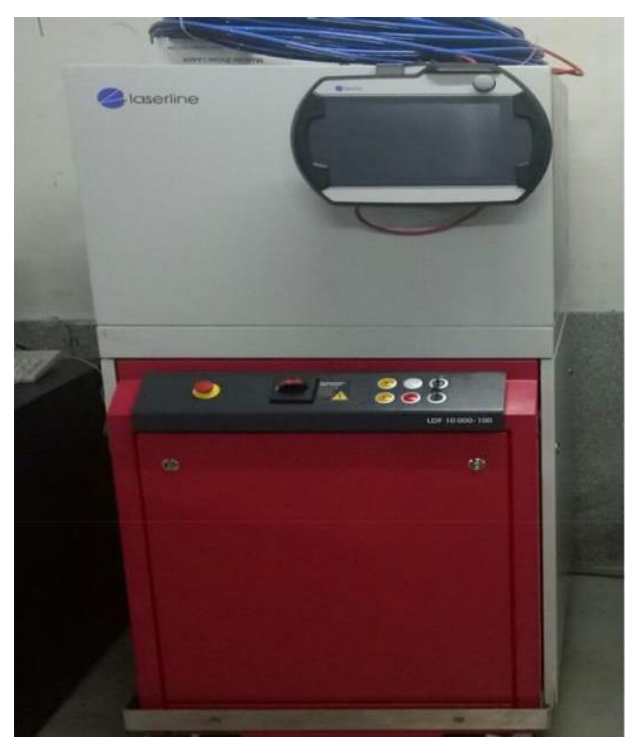

Fig 7. 10KW high power diode laser

The 6-axis KR-60, KUKA robot shown in fig 4.5 is used to mount Laser hardening optics shown in fig 4.6. An optical fiber, Laser Light Cable is used to connect the HPDL to Laser hardening optics.A pyrometer helps in achieving a close loop control of laser surface hardening process. With pyrometer we get to fix the desired temperature needed to harden the component, set along with a lower and upper limit of temperature.

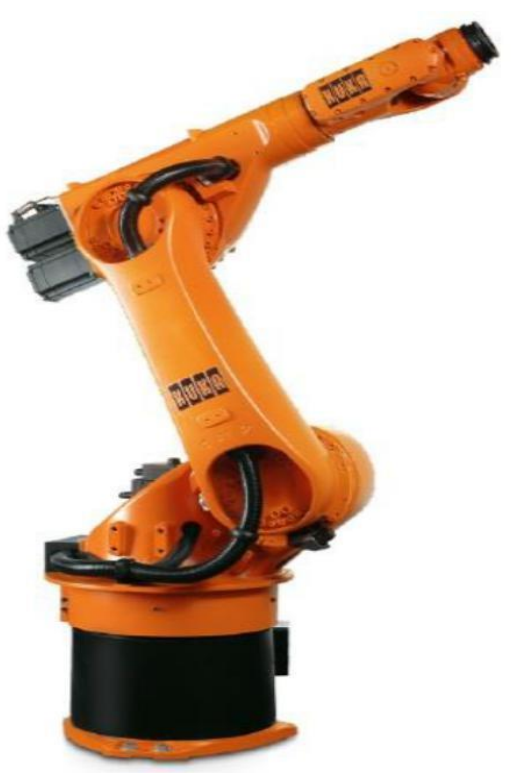

Fig 8. KR-60 Robot

\section{RESULTS AND DISCUSSIONS}

Data accumulation for Steam Turbine Blade of $\mathrm{X} 20 \mathrm{Cr} 13$ material is shown in table 1 below, showcasing the data under single table and helping us to find the optimum parameters.

\begin{tabular}{|l|c|c|}
\hline $\begin{array}{l}\text { Distance } \\
\text { from } \\
\text { surface } \\
\text { (mm) }\end{array}$ & $\begin{array}{l}\text { MicroVickers } \\
\text { Hardness for } \\
1 \mathrm{~kg} \mathrm{load}\end{array}$ & $\begin{array}{l}\text { Rockwell } \\
\text { Hardness } \\
\text { C scale } \\
\text { (HRC) }\end{array}$ \\
\hline 0.1 & 586.2 & 54 \\
\hline 0.2 & 580.7 & 54 \\
\hline 0.3 & 554.6 & 52 \\
\hline 0.4 & 552.8 & 52 \\
\hline 0.5 & 503.9 & 49 \\
\hline 0.6 & 492.6 & 48 \\
\hline 0.7 & 480.1 & 47 \\
\hline 0.8 & 453.3 & 45 \\
\hline 0.9 & 451.7 & 46 \\
\hline 1.0 & 462.6 & 45 \\
\hline
\end{tabular}

Table 1 Hardness results for X20Cr13 material

\begin{tabular}{|c|c|c|}
\hline $\begin{array}{l}\text { Distance from } \\
\text { surface (mm) }\end{array}$ & $\begin{array}{l}\text { Micro Vickers } \\
\text { Hardness for 1kg } \\
\text { load }\end{array}$ & $\begin{array}{l}\text { Rockwell } \\
\text { Hardness C } \\
\text { scale in HRC }\end{array}$ \\
\hline 0.1 & 728.4 & 61 \\
\hline 0.2 & 748.6 & 62 \\
\hline 0.3 & 759.4 & 62 \\
\hline 0.4 & 738.7 & 61 \\
\hline 0.5 & 747.8 & 61 \\
\hline 0.6 & 729.8 & 49 \\
\hline 0.7 & 535.8 & 52 \\
\hline 0.8 & 579.6 & 29 \\
\hline 0.9 & 296.5 & 29 \\
\hline 1.0 & 295.4 & 62 \\
\hline
\end{tabular}


Table 2. Hardness results for X20Cr13 material

\begin{tabular}{|c|c|c|}
\hline $\begin{array}{l}\text { Distance } \\
\text { from surface } \\
(\mathrm{mm})\end{array}$ & $\begin{array}{l}\text { Micro Vickers } \\
\text { Hardness for 1kg } \\
\text { load }\end{array}$ & $\begin{array}{l}\text { Rockwell } \\
\text { Hardness C scale } \\
\text { in HRC }\end{array}$ \\
\hline 0.1 & 728.4 & 61 \\
\hline 0.2 & 748.6 & 62 \\
\hline 0.3 & 759.4 & 61 \\
\hline 0.4 & 738.7 & 62 \\
\hline 0.5 & 747.8 & 61 \\
\hline 0.6 & 729.8 & 49 \\
\hline 0.7 & 535.8 & 52 \\
\hline 0.8 & 579.6 & 29 \\
\hline 0.9 & 296.5 & 29 \\
\hline 1.0 & 295.4 & 62 \\
\hline
\end{tabular}

Table 3. Hardness results for Non Malleable cast iron material

\begin{tabular}{|c|c|c|}
\hline $\begin{array}{l}\text { Distance from } \\
\text { surface } \\
(\mathrm{mm})\end{array}$ & $\begin{array}{l}\text { Micro Vickers } \\
\text { Hardness for } \\
\text { 1kg load }\end{array}$ & $\begin{array}{l}\text { Rockwell } \\
\text { Hardness C } \\
\text { scale in HRC }\end{array}$ \\
\hline 0.1 & 728.4 & 61 \\
\hline 0.2 & 748.6 & 62 \\
\hline 0.3 & 759.4 & 62 \\
\hline 0.4 & 738.7 & 61 \\
\hline 0.5 & 747.8 & 62 \\
\hline 0.6 & 729.8 & 61 \\
\hline 0.7 & 535.8 & 49 \\
\hline 0.8 & 579.6 & 52 \\
\hline 0.9 & 296.5 & 29 \\
\hline 1.0 & 295.4 & 29 \\
\hline
\end{tabular}

Table 4. Hardness results for carbon steel materials

\section{OBSERVATIONS}

For a same material and same set of laser surface hardening parameters, with increase in set temperature, the case depth increases. But increase in the set temperature also increases the laser power consumed. On the other hand with decrease in set temperature, the case depth decreases along with the decrease in laser power consumption

$>$.For a same material and same set of laser surface hardening parameters, with increase in scanning speed, the case depth decreases along with decrease in laser power consumption. On the other hand with decrease in scanning speed, the case depth increases along with increase in laser power consumption.

For a same material and same set of laser surface hardening parameters, with the decrease in spot size of focused laser beam the laser power consumption decreases. On the other hand with increase in spot size of focused laser beam the laser power consumption increases.

The same kind of material with a shiny surface that is, with more reflective surface consumes more laser power than a less or non-reflective surface for same set laser surface hardening parameter. This is because the shiny surface reflects major portion of laser beam focused on to it.

> The thickness of material, shape and size of the component also affects the laser surface hardening parameters. That is for same kind of material and same set of laser surface hardening parameters, different size and shape and different thickness of material region to surface be surface hardened can end up with different hardness results. 


\section{CONCLUSION}

A $10 \mathrm{KW}$ High Power Diode Laser with a 6-axis robotic arm was used to improve surface properties of X20Cr13 Steam Turbine Blade, Non-malleable Cast Iron Retainer and Carbon Steel Hub. The main objective was to reach hardness greater than 45 HRC till a case depth of at least $0.5 \mathrm{~mm}$ which can withstand the wear and tear and help in increasing life of a component. From Micro Vickers testing it was found that hardness of X20Cr13 material Steam Turbine Blade which had a hardness of 28 HRC prior to laser surface hardening increased to 49-54 HRC till a case depth of $0.5 \mathrm{~mm}$ after laser surface hardening. Non-malleable Cast Iron material Retainer with an initial hardness of 26 HRC developed a hardness of 61-62 HRC till a case depth of $0.5 \mathrm{~mm}$. similarly Carbon Steel material Hub with an initial hardness of 24 HRC developed a hardness of 61-62 HRC till a case depth of $0.5 \mathrm{~mm}$.

For a set temperature greater than $1350^{\circ} \mathrm{C}$ resulted in surface melting and for a set temperature below $1150^{\circ} \mathrm{C}$ no surface modification occurred for all three materials. The optimum process parameters for X20Cr13 material turbine blade was found out to be $1250^{\circ} \mathrm{C}$ and scanning speed of $400 \mathrm{~mm} / \mathrm{min}$ which produced a case depth till $1.2 \mathrm{~mm}$. Similarly the optimum process parameters for Non-malleable Cast Iron material Retainer was found out to be $1250^{\circ} \mathrm{C}$ and scanning speed of $900 \mathrm{~mm} / \mathrm{min}$ which produced a case depth till $1.1 \mathrm{~mm}$ and the optimum process parameters for Carbon Steel material Hub was found out to be $1250^{\circ} \mathrm{C}$ and scanning speed of $900 \mathrm{~mm} / \mathrm{min}$ which produced a case depth till $1.3 \mathrm{~mm}$.

That is the hardness of the surface increased by a factor of 1.8, 2.35 and 2.5 the initial surface hardness prior to laser surface hardening in X20Cr13, Nonmalleable Cast Iron and Carbon Steel Material respectively after laser surface hardening.

\section{REFERENCES}

[1]. S. Balasubramanian, V. Muthukumaran and p.Satyabalan, "A Study on the effect of process parameters of laser hardening in carbon steels." [2017]

[2]. Babic M, Balic J, Milfelner M, Belic I, Kokol P, Zorman N, Panjan P, "Robot laser Hardening and the problem of Overlapping laser Beam."[2013]

[3]. Fritz Klocke, Martin Schulz and Stephen Grafe, "Optimisation of the laser Hardening process by adapting the intensity distribution to generate a top-hat temperature distribution using freedom optics."[2017]

[4]. NguyenHung Improved Formability of Aluminum Alloys using Laser induced Hardening of Tailored Heat Treated Blanks.

[5]. MatejBabic, MatjaMilfelner, Igor Beli, Peter Problems Associated with a Robot Laser Cell used for Hardening by varying angle.

[6]. AshishKumarNath,A Theoretical and experimental study on laser surface hardening by repetitive laser pulses. [2011].

[7]. C. Soriano, J. Leunda, J. Lambarri, V. GarcíaNavas, C. Effect of laser surface hardening on the microstructure, hardness and residual stresses of austempered ductile iron grades.

[8]. Zhaoyun Ch Microstructure and hardness investigation of $17-4 \mathrm{PH}$ stainless steel by laser quenching.

[9]. H. Ki and S. So, "Optics and laser Technology process map for laser heat treatment of carbon steels," Opt. Laser Technol., Vol.44, No.7, pp.2106-2114, [2012]. 